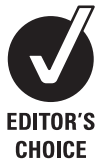

School of Human Movement and Sport Sciences, University of Ballarat, Ballarat, Australia

\section{Correspondence to}

Dr Natalie Saunders, School of Human Movement and Sport Sciences, University of Ballarat, P0 Box 663, Ballarat 3350 Australia;

n.saunders@ballarat.edu.au.

Accepted 17 March 2010 Published Online First 11 June 2010

\title{
Coaches' perspectives on implementing an evidence-informed injury prevention programme in junior community netball
}

\author{
N Saunders, L Otago, M Romiti, A Donaldson, P White, CF Finch
}

Objective For effective sports injury prevention, information is needed about the implementation context for interventions. This study describes coaches' feedback on the implementation of an evidence-informed injury prevention programme in community junior netball using coaches' perceptions and the RE-AIM framework. Methods A lower-limb injury prevention programme (Down to Earth; D2E), for teaching safe-landing techniques, was delivered to 31 coaches from 31 junior community netball teams in a 1-h workshop. Coaches then delivered a 6-week programme at team training sessions starting in the week before the competition season commenced. $65 \%$ of coaches completed a feedback survey 17 weeks after they had delivered the programme.

Results Most (88\%) coaches believed that D2E improved their players' ability to perform correct landing techniques in games and that players had retained these improvements over the season. The majority $(83 \%)$ indicated that an improvement in player athletic attributes was the greatest advantage of D2E, followed by a reduction in injury risk. Identified barriers to implementing D2E were running out of time and very young players finding the drills too difficult. Coaches reported that they needed more ideas for training drills that could be incorporated into their programmes and believed that their own coaching training did not adequately prepare them to implement an injury prevention programme.

Conclusions Although coaches believed that D2E was effective in developing correct landing techniques, some modifications are needed to make it more suitable for younger players and coach education by accreditation courses could be improved to support the implementation of injury prevention programmes.

To reduce injury rates ${ }^{1-3}$ or to modify risk factors associated with particular injury mechanisms, ${ }^{4-6}$ specific training programmes have been implemented. While such programmes currently exist, very few have been implemented with junior players of team sports for whom the rate and impact of injury is high. ${ }^{7-9}$ Given the importance of coaches in delivering training programmes and other safety initiatives to players, ${ }^{10} 11$ it is surprising that there have been very few studies of their injury prevention practices, safety promotion attitudes and ongoing role in implementing injury prevention programmes. ${ }^{12} 13$ Although coaches may not always have direct access to the latest injury prevention research evidence to inform their coaching practices, they do support implementing specific training programmes that can improve both player performance and reduce the risk of injury. ${ }^{12}$
Coaches play a key role in implementing game development strategies that prevent players being exposed to inappropriate injury risks as they progress through age levels of competition. ${ }^{14}$ However, a survey of 35 coaches in junior rugby union identified a clear need for further education about the mechanisms and early management of sports injury. ${ }^{15}$ Coach education within netball and soccer has recently been shown to be effective in helping to decrease the likelihood of injuries in community players. ${ }^{13}$ To our knowledge, however, the specific implementation issues of an injury prevention programme, from the perspective of coaches in junior sports, have not been published in the peer-review literature.

There has been recent interest in understanding the context in which sports injury prevention interventions are implemented in an effort to enhance the likelihood that they will be adopted and sustained by the target audience..$^{1617}$ One framework for investigating intervention effectiveness in the implementation context is RE-AIM. ${ }^{18} 19$ In the context of a training programme to lead players through specific exercises for injury prevention, the components of this framework translate to assessing the: cohort of coaches and players exposed to the programme (Reach); coaches' opinions of the advantages of the programme (Effectiveness); coaches' willingness to use the programme (Adoption); coaches' ability to implement the programme and adapt it to suit the needs of the players (Implementation) and the likelihood that coaches would continue to implement the programme (Maintenance).

This study considers the implementation of a training intervention specifically designed to prevent lower-limb injuries in netball. Netball has a high incidence of lower-limb injuries with ankle and knee injuries accounting for $30-84 \%$ and $8-41 \%$ of all injuries in adults, respectively. ${ }^{20-23}$ Although fewer lower-limb injuries occur in junior players, ${ }^{24}$ the incidence of injuries from falls $(35 \%)$ suggests that such injuries could be due to poor balance or a lack of other key movement skills. ${ }^{25} 26$ Incorrect landing is a frequent cause of lowerlimb injury in netball ${ }^{20} 2127$ and most of these injuries occur without direct contact with another player. ${ }^{21}$ This implies an underlying mechanism of intrinsic dysfunction in fundamental movement patterns that could be addressed through targeted training interventions.

This paper reports coaches' perceptions of, and suggestions for, improving the implementation of an evidence-informed safe-landing techniques 
programme. Down to Earth (D2E) is a netball-specific landing programme developed for community-level netball that specifically targets lower-limb injury prevention ${ }^{28}$ (Programme details are available from http://www.ballarat.edu.au/ard/ hmss/). D2E includes guidelines for safe-landing techniques, instructions for providing feedback and progressive exercises with specified sets and repetitions, to be completed over a 6 -week period. The efficacy of D2E has previously been quantified in terms of neuromuscular and biomechanical changes concurrent with a reduction in risk factors associated with lower-limb, particularly anterior cruciate ligament, injuries. ${ }^{28}$

This study fundamentally aimed to enhance the understanding of translating scientific evidence into practice by examining the perceived effectiveness of the D2E programme in 'real world' environments and assessing coaches' perspectives of the D2E programme in an implementation context, including the appropriateness for junior players. The specific outcomes of this study are intended to inform the future implementation of the D2E programme to a broader audience.

\section{METHODS}

This study was a component of a larger controlled ecological assessment of a netball-specific coach education programme aimed at educating and empowering netball coaches to deliver $\mathrm{D} 2 \mathrm{E}$ to junior players. This study has been conducted using the RE-AIM evaluation framework. ${ }^{19} 29$ Table 1 lists how each dimension of this framework has been defined in the context of this study.

Affiliated clubs from a Victorian netball association were recruited before the 2007/8 summer competition. Clubnominated teams from the 11\& under (U11), 13\& under (U13) and 15\& under (U15) competitions were invited to participate in the study. Feedback obtained from team coaches about the benefits and challenges of implementing the D2E programme with their players, as assessed at the end of the playing season (17 weeks post- programme implementation) provides the basis for the results presented. The University of Ballarat Human Research Ethics Committee approved this study.

During the preseason, 31 coaches from 31 teams attended a 1-h coach education session (D2E workshop). The rationale for a 6 -week D2E programme for players was outlined and the

Table 1 Application of the RE-AIM framework in the evaluation of the D2E programme among coaches of junior netball teams

\begin{tabular}{ll}
\hline RE-AIM dimension & Evaluation measure \\
\hline Reach & The exposure of the programme to both coaches \\
of junior players and subsequently junior players, \\
within the association \\
\% of D2E coaches who believed that D2E was \\
effective in: \\
Effectiveness \\
Adoption \\
Coaches' responses on facilitating adoption of \\
D2E, specifically the most relevant age group and \\
skill level (figure 1) \\
Coaches' opinions on resources to improve \\
implementation \\
Factors or circumstances identified as challenges \\
for implementing D2E (table 3) \\
No of coaches providing constructive feedback \\
on D2E \\
No of coaches who intend to use D2E with players \\
in the future
\end{tabular}

D2E, Down to Earth.
D2E booklet was distributed. Specific exercises and coaching scenarios were demonstrated and coaches were encouraged to discuss anticipated barriers to implementing D2E and how to overcome them, particularly in relation to working with young children and the increasing difficulty of the training exercises. At the end of the D2E workshop, coaches were asked to implement D2E at team training sessions starting in the week before the first game of the season.

Coaches completed an end-of-season survey 17 weeks after they had completed the delivery of D2E to their players. The survey included 17 questions about this study; 12 multiple choice, four dichotomous (yes/no), and one open-ended question about the perceived advantages, disadvantages, barriers and facilitators regarding D2E. Coaches could indicate more than one response to the multiple-choice questions.

All data were precoded and double-entered into a Microsoft Access database. All data were checked and cleaned before being transferred to SPSS for analysis. As this is a descriptive study of coach views, all data are presented as a percentage of those coaches who responded to the end-of-season survey. Some direct quotes in response to the open-ended question are presented.

\section{RESULTS}

Compliance data were collected throughout the season for all 31 coaches to monitor the implementation of the D2E programme. While it is known that all 31 coaches did implement the 6 -week programme, only 24 coaches ( $77 \%$ response rate) completed the end-of-season survey. Results are therefore presented as a percentage of these 24 respondents. No information was collected about non-respondents to the survey. Table 1 shows the D2E evaluation measures against the relevant RE-AIM dimensions.

While results presented are based on 24 coaches, the actual reach of the programme was greater. Fifty per cent of coaches of junior players in the association $(n=31)$ agreed to participate in the study and implemented the 6-week programme. Subsequently, 248 junior players were exposed to D2E by these coaches, which accounts for approximately $50 \%$ of the junior players who participated in the 2007/8 summer competition draw.

When asked if players' ability to perform correct landing techniques in games improved while completing D2E, $88 \%$ of coaches concurred. These coaches also believed that their players had retained these improvements throughout the season. The coaches' responses to the perceived programme effectiveness in contributing to reducing the players' risk of ankle and knee injuries ranged from extremely effective (17\%), quite effective (54\%), slightly effective $(21 \%)$ to neither being effective or ineffective (8\%).

In addition, coaches were asked specifically to indicate the advantages of players completing D2E. The majority nominated improvement in player athletic attributes, followed by reduced injury risk and learning correct landing techniques (table 2). Three barriers to implementing D2E (given by $45 \%$ of coaches) were running out of time (42\%), players finding the drills too difficult (42\%) and too many sets and repetitions for young players to complete (38\%). The coaches reported modifying the programme drills to overcome these barriers by decreasing the number of sets and repetitions (60\%) and making the drills easier (45\%).

Most coaches indicated that they would use D2E with all ages and ability levels, although they identified U13, U15 and low-skilled players as those who would benefit most from the programme (figure 1). 
The majority of coaches $(92 \%)$ indicated that an information booklet is valuable in making it easier to implement D2E. Most suggested that specific coach training courses (71\%) and including safe-landing exercises in all team training sessions (79\%) would also improve implementation. A training manual/book was identified by $96 \%$ of coaches as an important additional resource for helping them to implement D2E. A D2E information kit (79\%) and website (71\%) were common suggestions for additional coach resources. Incorporating D2E training into existing coach training workshops (96\%) and/or coaching accreditation courses (83\%) were considered to be the best way to encourage coaches to use D2E.

Factors or circumstances that were identified as making D2E harder for coaches to implement are presented in table 3. Most coaches (79\%) indicated that they require help to develop or modify training drills to incorporate D2E into their training sessions. They also reported $(42 \%)$ that their general coach training did not adequately prepare them to implement a safe-landing programme such as D2E. Other implementation barriers were related to the challenges of working with young players, including poor concentration and motivation levels (83\%) and the perceived value of injury prevention programmes by junior players $(71 \%)$.

Finally, coaches were invited to provide comments and ideas on how to improve the implementation of future injury prevention interventions. Comments included:

Congratulations... The earlier the better that we as coaches introduce 'Down to earth'.

Fantastic concept. I have learnt a lot. My team enjoyed it. They were a team of limited skills and I think they got some benefit from it. They enjoyed it for the fun but found it hard to concentrate on the skills bit.

A number of comments indicated that D2E could be improved through the modification of repetitions and the degree of skill required for each exercise:

Have separate training booklets for juniors and more advanced booklets for older girls so they don't get bored.

Well set up programme but struggled teaching to under $11 \mathrm{~s}$ because they lost focus and became bored. Drills were either too repetitive or out of their depth for them complete but it is important to add safe landing techniques. Booklet well explained.

Other comments referred to different aspects of implementing the programme and how it could be better incorporated into player training sessions or brought into coaching culture. These comments demonstrate that, to be effective, D2E needs

Table 2 Coaches' views about the advantages of completing the D2E programme

\begin{tabular}{lc}
\hline Response to question as stated on survey & $\begin{array}{c}\text { \% of responding } \\
\text { coaches' (n=24) }\end{array}$ \\
\hline $\begin{array}{l}\text { Players improved other athletic attributes (strength, balance, } \\
\text { coordination, flexibility, etc) }\end{array}$ & 83 \\
$\begin{array}{l}\text { Reduced risk of injury (particularly to the knee and/or ankle) } \\
\text { Junior players learn correct landing technique right from the } \\
\text { outset }\end{array}$ & 79 \\
$\begin{array}{l}\text { Improved ability to avoid stepping rule violations } \\
\text { Players improved other game skills (catching, passing, game }\end{array}$ & 63 \\
sense, etc) & 50 \\
\hline
\end{tabular}

D2E, Down to Earth. to be incorporated into regular training sessions, rather than as a stand-alone programme. Comments included:

$\mathrm{D} 2 \mathrm{E}$ training needs to be incorporated into normal training sessions and be age/skill specific.

As many practical demonstrations and training workshops as possible... delivered by qualified personnel.

Finally, coaches were asked if they intended to use D2E in the future with players of the same age and playing level as those in their current team/s. The majority of coaches (88\%) indicated that they would continue to implement D2E.

\section{DISCUSSION}

D2E has previously been shown to be efficacious in reducing risk factors associated with lower-limb injury. ${ }^{28}$ The reach of the current study expanded from the original efficacy study. ${ }^{28}$ Using coach training as a delivery platform; a key factor for effective translation of scientific evidence into practice, ${ }^{12}{ }^{13}$ the capacity to reach a large number of junior community netball players that could not be reached otherwise was enhanced. In total, approximately half of junior players and coaches of junior players from the target age groups within an association were exposed to D2E.

Coaches' perspectives about the effectiveness of D2E indicated that the majority believed that it was effective in teaching junior players correct landing techniques and reducing their lower-limb injury risk. It is important to acknowledge that this implies that coaches were observing landing techniques that better matched D2E information and subsequently injury risk was reduced. In their opinion, D2E was also effective in improving players' athletic attributes and improving game-related skills, such as passing, catching and abiding by the stepping rule.

To maximise D2E programme adoption, the current implementation strategy aimed to empower and educate coaches in the principles of safe-landing technique training, using the D2E resource. The coaches were then expected to implement the programme with their players while noting any emerging challenges. Although coaches adopted the programme, they were more likely to consider D2E relevant for U13, U15 and less skilled players than the very young or more skilled, senior players. At times, the programme was found to be too difficult

Table 3 Coaches' perceptions of the factors or circumstances that made it harder for coaches to implement the D2E programme as part of every junior netball training session (\% of 24 respondents)

\begin{tabular}{lc}
\hline Response to question & $\begin{array}{r}\text { \% of responding } \\
\text { coaches (n=24) }\end{array}$ \\
\hline Coach-related factors & \\
Lack of: & 79 \\
Ideas for training drills & 42 \\
Skills/not being trained properly in this aspect of coaching & 63 \\
Time at training & 36 \\
Coaching skills & 33 \\
Training space & 21 \\
Training equipment & \\
Player-related factors & 83 \\
Players not listening/lack of motivation & 71 \\
Older players thinking they do not need it & 71 \\
Players not attending training regularly & 46 \\
Training drills are boring & 8 \\
Other & \\
\hline
\end{tabular}

D2E, Down to Earth. 


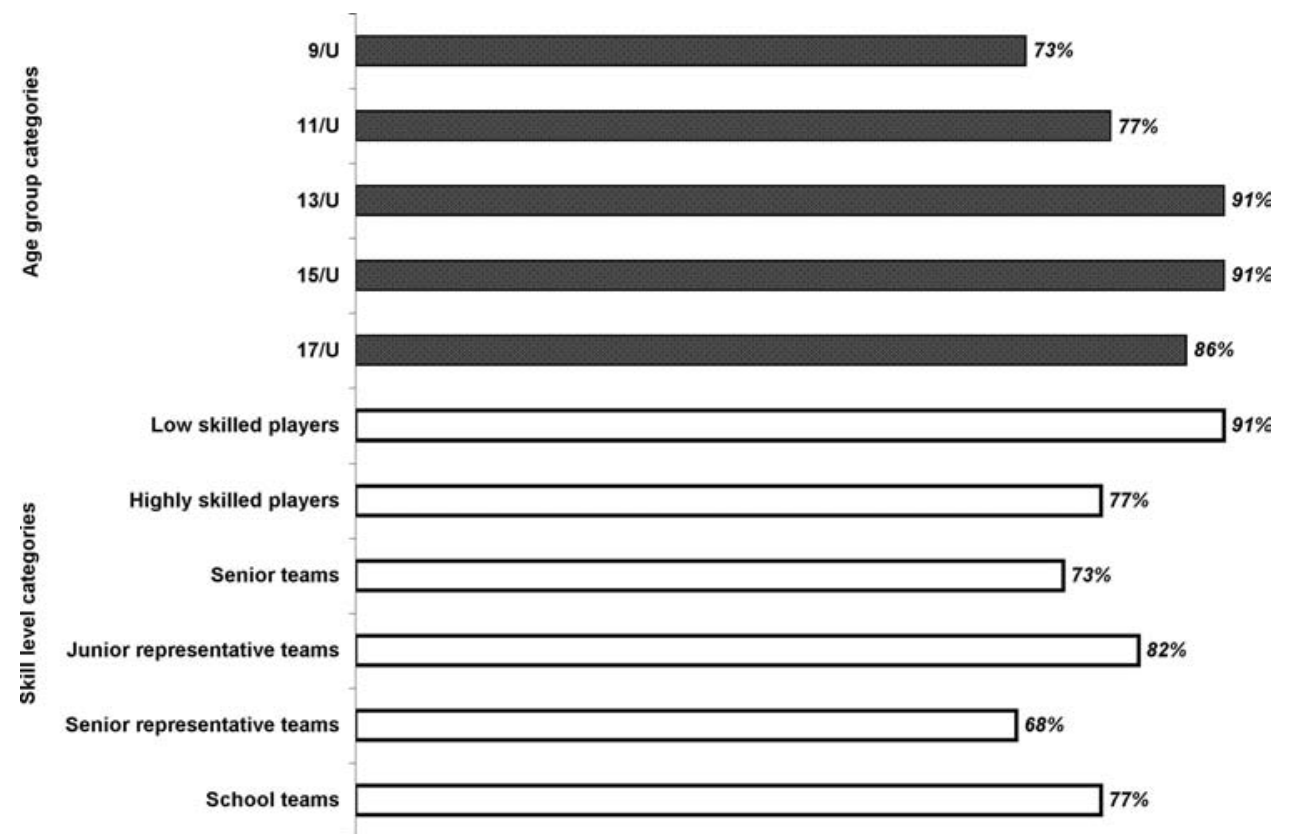

Figure 1 Coaches' beliefs as to the age group and skill level Down to Earth is most relevant for (\% of the 24 respondents).

and boring for the younger players. In contrast, there was the perception that older players lacked motivation and did not believe they needed to develop a safe-landing technique.

In support of existing research ${ }^{12} 13$ it seems that incorporating D2E into existing coaching workshops and/or accreditation courses would be the most appropriate way to encourage coaches to implement the programme. This would facilitate the dissemination of critical information about correct landing techniques, motor learning concepts and how to access resources, thereby addressing some of the barriers identified to facilitate the adoption of D2E.

The D2E booklet was considered an important resource to assist coaches to implement the programme, with indications that a website and information kit would further aid implementation. Coaches also felt that specific coach training within existing accreditation schemes would assist programme implementation. This finding reinforces coaches' views that they need to acquire adequate knowledge to maximise their capacity to implement injury prevention strategies. ${ }^{13}$ Coaches also identified a range of barriers to the implementation of $\mathrm{D} 2 \mathrm{E}$ including a lack of skills/not being trained properly in this aspect of coaching. While this finding supports the importance of developing the coach as the interface between efficacy and effectiveness in sports injury prevention, further investigation is required to ascertain the particular aspects of the programme for which coaches lacked skills and understanding. In addition, D2E incorporates three key aspects: guidelines for safe and effective landing; a decreasing feedback schedule to enable players eventually to correct their own technique independently and a set of drills to reinforce these components. ${ }^{28}$ The programme focuses on the control of single-leg landings that are common in netball and uses a progression of drills to develop the level of control required. ${ }^{28}$ It would be important for future research to ascertain whether a single component of the programme, or a combination, was more difficult for the coaches to implement with particular types of players.

The coaches identified how they adapted and modified D2E to facilitate implementation in their own specific coaching environment. Strategies included decreasing the number of sets and repetitions for exercises and making the exercises easier for younger players, although this was sometimes difficult for coaches to do. This highlights a problem with the translation of scientific evidence generated from the laboratory into a realworld environment. In the case of D2E, the adaptation and modification by coaches makes it difficult to know if the programme would still be effective if a different set of activities were implemented. A comparison of injury rates, however, between a cohort of players exposed to D2E and a matched control group could shed light on the effect of programme modification.

Player-related factors would also need to be addressed before further implementation of D2E. Player concentration and motivation levels, and their perceived value of injury prevention programmes, are key factors that would need consideration. Although our coaches heavily promoted this safe-landing programme, coaches reported that player perceptions of injury prevention were somewhat negative. Subsequently, the only reported disadvantage of implementing D2E was player boredom. Coaches believed that a wider range of drills and exercises, relevant to the skill/age level of players, would enable coaches to deliver D2E in a more enjoyable and game-like way. In addition, coaches believed that players would be engaging in injury prevention practice without consciously being aware of it. Alternatively, a performance-enhancing benefit has been identified by coaches ${ }^{12}$ and players $^{5}$ as a factor that would be likely to enhance the adoption of an injury prevention programme. Future injury prevention intervention research, particularly in netball, should thus consider the context in which the programme is promoted.

Evaluation of the maintenance of an intervention should include an assessment of its sustainability over time, particularly after the cessation of the formal research phase. ${ }^{19}$ This assessment of D2E maintenance was based on coaches' perceptions 17 weeks post-implementation, allowing coaches time to reflect on and observe the intermediate outcomes of the programme. Most coaches stated that they would implement D2E in the future with players of most ages and skill levels, suggesting that 


\section{What is already known on this topic}

- Specific training programmes have been successful in reducing injury rates or modifying risk factors associated with injury mechanisms.

- The coach and using coach training as a delivery platform is a key factor for the effective translation of scientific evidence into practice.

\section{What this study adds}

- This study evaluates an injury prevention intervention within its intended implementation context.

- Key barriers were identified towards the implementation of an injury prevention programme and strategies to overcome these to enhance our understanding of translating scientific evidence into practice within a junior sport community.

they were willing to maintain programme implementation well into the future. It would be worthwhile to reassess the retention of coaches' knowledge and beliefs of D2E principles with an extended follow-up (eg, into the next season).

A major strength of this study is the theoretical underpinning of the evaluation. The RE-AIM framework ${ }^{19}$ is a well recognised framework for effectiveness research, and this study is one of the few to address the final stages of the translating research into injury prevention practice framework ${ }^{16}$ by considering an injury prevention intervention within its intended implementation context. Although this study was restricted to one netball association, and on a relatively small sample of coaches, there is no reason to believe that the issues raised would be very different to those raised by junior coaches elsewhere.

In summary, the effectiveness of D2E has been structured and evaluated using the RE-AIM framework, by examining the perceptions of coaches at 17 weeks post-intervention. The programme reached a large proportion of the coaches and players who were targeted and coaches believed the programme to be effective in improving their players' ability to perform correct landing techniques and improving players' athletic attributes. Barriers towards the adoption and implementation of $\mathrm{D} 2 \mathrm{E}$ were identified, as were potential strategies to overcome them including: incorporating the programme into coaching accreditation courses; providing more ideas for training drills; making the exercises and drills less physically demanding and more fun and game-like and developing and disseminating a range of support resources. Fundamentally, this research has enhanced the understanding of translating scientific evidence into practice within a junior sport community.

Funding This study was funded by the Commonwealth Department of Health and Ageing, Sport and Recreation Victoria. Caroline Finch was supported by an NHMRC Principal Research Fellowship.

Competing interests None.

Contributors Smartplay Victoria, Netball Victoria, Netball Australia, Ballarat Netball Association, Altona Netball Association.

Ethics approval This study was conducted with the approval of the University of Ballarat Human Research Ethics Committee.

Provenance and peer review Not commissioned; externally peer reviewed.

\section{REFERENCES}

1. Myklebust G, Engebretsen L, Braekken IH, et al. Prevention of anterior cruciate ligament injuries in female team handball players: a prospective intervention study over three seasons. Clin J Sport Med 2003;13:71-8.

2. Hewett TE, Lindenfeld TN, Riccobene JV, et al. The effect of neuromuscular training on the incidence of knee injury in female athletes. A prospective study. Am J Sports Med 1999;27:699-706.

3. Caraffa A, Cerulli G, Projetti M, et al. Prevention of anterior cruciate ligament injuries in soccer. A prospective controlled study of proprioceptive training. Knee Surg Sports Traumatol Arthrosc 1996;4:19-21.

4. Chimera NJ, Swanik KA, Swanik CB, et al. Effects of plyometric training on muscle-activation strategies and performance in female athletes. J Athl Train 2004;39:24-31.

5. Myer GD, Ford KR, Palumbo JP, et al. Neuromuscular training improves performance and lower-extremity biomechanics in female athletes. J Strength Cond Res 2005; 19:51-60.

6. Lephart SM, Abt JP, Ferris CM, et al. Neuromuscular and biomechanical characteristic changes in high school athletes: a plyometric versus basic resistance program. Br J Sports Med 2005;39:932-8.

7. Emery CA, Meeuwisse WH, McAllister JR. Survey of sport participation and sport injury in Calgary and area high schools. Clin J Sport Med 2006;16:20-6.

8. Caine D, Maffulli N, Caine C. Epidemiology of injury in child and adolescent sports: injury rates, risk factors, and prevention. Clin Sports Med 2008;27: 19-50, vii.

9. Finch C, Cassell E. The public health impact of injury during sport and active recreation. J Sci Med Sport 2006;9:490-7.

10. Chalmers DJ, Simpson JC, Depree R. Tackling rugby injury: lessons learned from the implementation of a five-year sports injury prevention program. J Sci Med Sport 2004;7:74-84.

11. Finch C, McGrath A. SportSafe Australia: a national sports safety framework A report prepared for the Australian Sports Injury Prevention Taskforce. Government report. Canberra: Australian Sports Commission, 1997.

12. Twomey D, Finch C, Roediger E, et al. Preventing lower limb injuries: is the latest evidence being translated into the football field? J Sci Med Sport 2009;12:452-6.

13. Gianotti S, Hume PA, Tunstall H. Efficacy of injury prevention related coach education within netball and soccer. J Sci Med Sport 2010;13:32-5.

14. Romiti M, Finch CF, Gabbe B. A prospective cohort study of the incidence of injuries among junior Australian football players: evidence for an effect of playing-age level. Br J Sports Med 2008;42:441-6.

15. Carter AF, Muller R. A survey of injury knowledge and technical needs of junior Rugby Union coaches in Townsville (North Queensland). J Sci Med Sport 2008;11:167-73.

16. Finch C. A new framework for research leading to sports injury prevention. J Sci Med Sport 2006;9:3-9

17. Van Tiggelen D, Wickes S, Stevens V, et al. Effective prevention of sports injuries: a model integrating efficacy, efficiency, compliance and risk-taking behaviour. Br J Sports Med 2008;42:648-52.

18. Finch CF, Donaldson A. A sports setting matrix for understanding the implementation context for community sport. Br J Sports Med. Published Online First: 21 January 2009. doi: 10.1136/bjsm.2008.055798.

19. Glasgow RE, Vogt TM, Boles SM. Evaluating the public health impact of health promotion interventions: the RE-AIM framework. Am J Public Health 1999;89:1322-7

20. Hopper D, Elliott B, Lalor J. A descriptive epidemiology of netball injuries during competition: a five year study. Br J Sports Med 1995;29:223-8.

21. McKay GD, Payne WR, Goldie PA, et al. A comparison of the injuries sustained by female basketball and netball players. Aust J Sci Med Sport 1996;28:12-17.

22. $\mathbf{0 t a g o} \mathbf{L}$, Peake J. The role of insurance data in setting priorities for netball injury prevention strategies. J Sci Med Sport 2007;10:105-9.

23. McManus A, Stevenson MR, Finch CF. Incidence and risk factors for injury in non-elite netball. J Sci Med Sport 2006;9:119-24.

24. Finch C, Valuri G, Ozanne-Smith J. Sport and active recreation injuries in Australia: evidence from emergency department presentations. Br J Sports Med 1998;32:220-5.

25. Routley V, Ozanne-Smith J. Sport review injuries - an overview. Hazard 1991;8:1-6

26. Routley V. Sports injuries in children - the five most commonly presented sports Hazard 1991;:9:1-8.

27. $\mathbf{0 t a g o} \mathbf{L}$. A survey of knee injured netballers. Paper presented at the Australian Conference of Science and Medicine in Sport, Adelaide, 13-16 October 1998. Sports Medicine Australia.

28. Saunders $\mathbf{N}$. Characteristics of the female landing pattern. PhD Thesis, University of Ballarat, Ballarat, 2006.

29. Lowe J, Yang J, Heiden E, et al. Intervention research and program evaluation. In: Carlson GA, Sleet DA, DiClemente RJ, eds. Injury prevention and violence prevention: behavioural science theories, methods and applications. San Francisco, California, USA: John Wiley \& Sons, 2006:188-209. 